\section{Diamond planar refractive lenses for third- and fourth-generation X-ray sources}

\section{Bernd Nöhammer, ${ }^{a \star}$ Joanna Hoszowska, ${ }^{b}$ Andreas K. Freund $^{\mathrm{b}}$ and Christian David ${ }^{\mathrm{a}}$}

\author{
a Laboratory for Micro- and Nanotechnology, Paul Scherrer \\ Institute, $\mathrm{CH}-5232$ Villigen-PSI, Switzerland, and ${ }^{b}$ European \\ Synchrotron Radiation Facility, BP 220, F-38043 Grenoble \\ CEDEX, France. E-mail: bernd.noehammer@psi.ch
}

The fabrication and testing of planar refractive hard X-ray lenses made from bulk CVD diamond substrates is reported. The lens structures were generated by electron-beam lithography and transferred by reactive-ion etching into the diamond. Various lens designs were fabricated and tested at 12.4 and $17.5 \mathrm{keV}$ photon energy. Efficiencies of up to $71 \%$ and gains of up to 26 were achieved. A line focus of $3.2 \mu \mathrm{m}$ (FWHM) was measured. These lenses should be able to withstand the extreme flux densities expected at the planned fourth-generation X-ray sources.

Keywords: X-ray optics; focusing; X-ray free-electron lasers; reactive-ion etching.

\section{Introduction}

High-brilliance third-generation synchrotron X-ray sources have proven to be outstanding tools for the study of condensed matter (Laclare, 2001). While new storage-ring sources of this type are being commissioned, under construction or planned, a new fourth-generation of X-ray sources based on self-amplification of spontaneous emission (SASE) inside a long undulator placed in a linear accelerator is being studied (Hasnain et al., 2001). These sources are expected to give extremely intense ultra-short pulses of highly coherent radiation with a peak brilliance of up to ten orders of magnitude above today's third-generation synchrotron sources (Sonntag, 2001). Such SASE-based free-electron laser (FEL) projects in Hamburg (Materlik \& Tschentscher, 2001) and Stanford (Nuhn et al., 2002) have been described very recently. Both types of sources have an extremely broad range of applications (Freund, 2001).

Amongst many other scientific and technological challenges, the development of suitable focusing X-ray optics capable of withstanding very intense radiation, which causes an extreme heat load and photodissociation of the lens material, is a major issue. This problem is of vital importance for the fourth-generation sources and has been addressed in many recent conferences, specific workshops and studies (Tatchyn et al., 1997, 2001; Mills et al., 2000; Materlik \& Tschentscher, 2001; Wootton et al., 2001). In this context, compound refractive lenses (CRLs) for X-rays, such as the lenses proposed by Tomie (1994, 1997, 1998) and those experimentally studied by Snigirev et al. (1996), may provide advantages compared with diffractive or reflective optics (Schroer, Tümmler et al., 2001; Pantell et al., 2001). First versions of these devices were produced by simply drilling holes into a low absorbing material, for example, aluminium or beryllium (Snigirev et al., 1996; Lengeler et al., 1998). For microfocusing applications the circular shape of the holes led to strong aberrations. More recently, an advanced technology has been developed to fabricate aluminium CRLs with surfaces close to the ideal parabolic shape and greatly improved performance (Lengeler $e t$ $a l ., 1999)$. These CRLs have been shown to work very well for X-ray energies above about $18 \mathrm{keV}$. For example, a gain exceeding three orders of magnitude and a spot size of $0.55 \times 5.5 \mu \mathrm{m}$ have been obtained at the ESRF at $19.5 \mathrm{keV}$ (Schroer, Lengeler et al., 2001; Lengeler et al., 2001). However, at medium and lower X-ray energies a lot of radiation is absorbed in these optical elements because of the relatively high atomic number of aluminium (Lund, 1997). Although recent results are very encouraging (Lengeler, 2002), the pressing technique that is used is not easily transferable to lens materials that are more suitable, such as $\mathrm{Be}, \mathrm{B}$ or $\mathrm{C}$.

Recently, a planar fabrication technology has been applied to obtain silicon devices with minimized absorption that are capable of focusing hard X-rays in one direction, i.e. to a line focus (Aristov et al., 2000; Snigireva et al., 2001). These refractive lenses are designed in such a way that passive parts of the lens material, i.e. parts that merely cause phase shifts of multiples of $2 \pi$, are removed. To obtain diffraction-limited focusing, the remaining parts of the lens have to be in phase. Consequently an optimum optical performance can only be expected for certain photon energies (each of them representing a different phase shift of some multiple of $2 \pi$ ). Two-dimensional focusing can be obtained if we use two such devices in series with orthogonal orientation (Aristov et al., 2001).

We used this approach to design planar refractive lenses made of diamond. Besides other materials like $\mathrm{Li}$ and Be (Bionta, 2000), diamond is one the best materials to meet the extreme requirements of an FEL source (Freund, 1995; Materlik \& Tschentscher, 2001; Wootton et al., 2001) because diamond has a very low absorption coefficient, high thermal conductivity, a low thermal expansion coefficient and thermal stability. Moreover, the geometry of planar refractive lenses provides efficient heat dissipation by the substrate. Therefore, the described combination of material and geometry appears to be a promising approach.

\section{Lens fabrication}

The planar refractive lenses were made from $200 \mu \mathrm{m}$-thick synthetic CVD diamond substrates by electron-beam lithography and reactiveion etching according to the fabrication steps illustrated in Fig. 1. For two purposes, we deposited a $1 \mu \mathrm{m}$-thick layer of chromium on the substrates. On one hand, it provided sufficient electrical conductivity to avoid electrostatic charging during the electron-beam lithography step, and, on the other hand, it served as a hard mask during the reactive-ion etching (RIE) into the diamond. The lens patterns were written into a $150 \mathrm{~nm}$-thick layer of electron-beam resist (Fig. 1a) and transferred into a $50 \mathrm{~nm}$-thick layer of aluminium by a lift-off process (Fig. 1b). The aluminium layer was used as a mask for the patterning of the chromium layer using a chlorine-based plasma etching process (Fig. 1c). The chromium structures can then be used as a hard mask in the deep reactive-ion etching of diamond by an oxygen plasma (Fig. 1d). We were able to obtain an etched depth of $40 \mu \mathrm{m}$, which was limited by erosion of the chromium mask.

Some examples of the resulting lenses can be seen in Fig. 2. The top micrograph shows a set of lenses each with an aperture of $100 \mu \mathrm{m}$. The curvature of the refracting surfaces increases from left to right, while the number of lenslets put in series decreases accordingly, so that the focal length of all five devices is $250 \mathrm{~mm}$ for $12.4 \mathrm{keV}$ and $500 \mathrm{~mm}$ for $17.5 \mathrm{keV}$ photon energy. A larger lens with an aperture of $500 \mu \mathrm{m}$ and a focal length of $500 \mathrm{~mm}$ and $1000 \mathrm{~mm}$ (for 12.4 and $17.5 \mathrm{keV}$, respectively) is shown in the centre image.

For the highest performance, the side walls of the etched structures must be vertical and smooth. The lower scanning-electron micrograph reveals the limitations of the deep etching process used. Although the lower half of the side wall appeared to be of good 
quality, the upper half showed increased roughness and a slope angle of about $80^{\circ}$. A kind of 'wrapping paper' structure can also be seen around the lenses, which causes a step of about $1 \mu \mathrm{m}$ between the upper and the lower part of the lens. These kinds of effects appeared in all our samples and can be explained by a lateral erosion of the chromium hard mask during the deep reactive-ion etching. Such phenomena are known in microfabrication technology as facetting [see, for example, Schutz (1988)] and can be reduced by optimization of the applied mask materials and process parameters.

\section{Measurements}

The diamond planar refractive lenses were tested at the Optics Beamline BM5 of the European Synchrotron Radiation Facility. Dipole radiation monochromated by a double-crystal Si(111) monochromator was used. The horizontal and vertical source sizes were $300 \mu \mathrm{m}$ and $80 \mu \mathrm{m}$ (FWHM), respectively. We oriented the lenses to focus in the vertical direction. For a lens-to-source distance of $40 \mathrm{~m}$ the geometrical image size of the source was approximately $0.5,1$ and $2 \mu \mathrm{m}$ for 250,500 and $1000 \mathrm{~mm}$ focal length, respectively. The efficiency of the lenses, i.e. the fraction of the incident radiation going into the focal spot, was measured by scanning a $20 \mu \mathrm{m}$-wide
Electron-beam lithography



(a)

Plasma etching of $\mathrm{Cr}$ hard mask

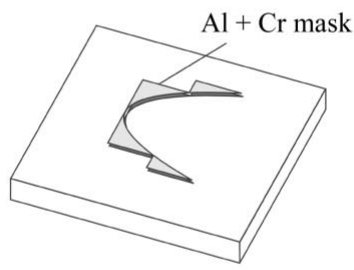

(c)
Evaporation of AL; lift-off

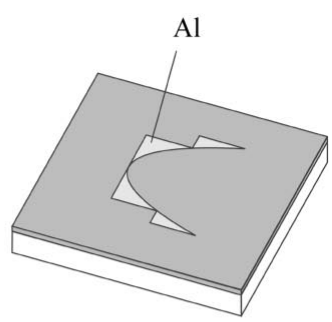

(b)

Deep reactive-ion etching of diamond

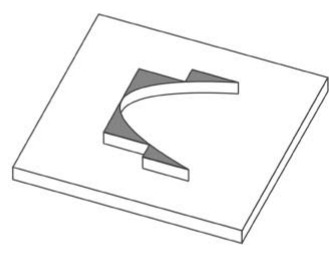

(d)

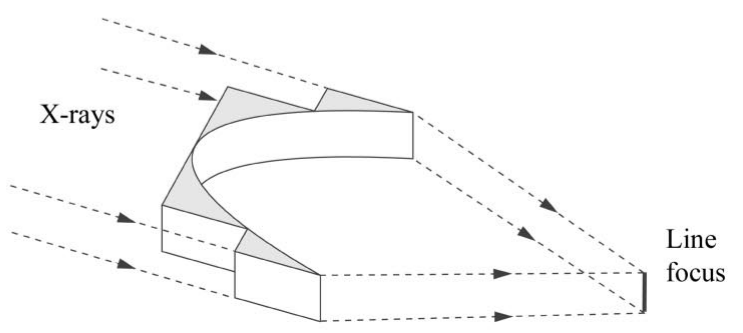

Figure 1

Top: fabrication process to generate the planar diamond refractive lenses ( $a-$ $d)$. Bottom: schematic sketch to illustrate the one-dimensional focusing by a planar refractive lens with minimized absorption (lower figure). vertical slit through the image plane of the lenses. The measured data were processed according to a routine previously developed for characterizing diffractive lenses (David \& Souvorov, 1999). We obtained a transmission of $78 \%$ for a $100 \mu \mathrm{m}$ aperture and $67 \%$ for a $500 \mu \mathrm{m}$ aperture, respectively, for $17.5 \mathrm{keV}$ photon energy. For lower energies a small decrease in transmission was observed $(72 \%$ for a $100 \mu \mathrm{m}$ aperture at $12.4 \mathrm{keV}$ ), which can be explained by higher absorption losses in the diamond sample. However, theoretical calculations have shown that absorption plays only a minor role. Taking into account photoelectric absorption and losses due to Compton scattering (Chantler, 1995) and making a numerical


Figure 2

Scanning electron micrographs of planar refractive lens structures etched $40 \mu \mathrm{m}$ deep into a bulk diamond substrate. See text for details. 
average of the transmission across the lens (see also Aristov et al., 2000), we found that the efficiency should be higher than $93 \%$ for all lens designs at $17.5 \mathrm{keV}$ photon energy. The main losses were supposedly caused by elastic scattering due to the polycrystalline structure of the diamond substrates and by scattering due to distortions and roughness of the lens surface. The former can be avoided by the use of single-crystal diamonds. The latter should be sufficiently decreased by the use of improved mask materials and process parameters.

To investigate the spatial resolving power of the lenses we scanned two crossed slits that formed a pinhole of size $2 \mu \mathrm{m} \times 0.5 \mu \mathrm{m}$ in the horizontal and vertical directions, respectively, through the image plane of the lenses. Fig. 3 shows a grey-tone plot of a two-dimensional intensity map of a lens with a $500 \mu \mathrm{m}$ aperture and $1 \mathrm{~m}$ focal length taken at $17.5 \mathrm{keV}$ photon energy.

The (horizontal) length of the line focus is about $15 \mu \mathrm{m}$, which is significantly less than the etch height of the lens $(40 \mu \mathrm{m})$. We believe that this mismatch occurs because the main contributions to the line focus originate from the lower high-quality part of the lens. The upper part has non-vertical side walls and therefore acts like a prism, which not only focuses in the vertical direction but also causes a deflection in the horizontal direction. This deflection leads to a faint elongation of the left part of the line focus, as can be seen in Fig. 3. In addition it is very likely that the sample's roughness (a few micrometers), which is caused by the RIE process, also plays a role, as this roughness can cause scattering (and therefore the loss) of parts of the X-ray beam close to the sample surface.

The vertical FWHM of the line focus (taken in the region with maximum intensity) was $3.2 \mu \mathrm{m}$. For an aberration-free lens this width is given by a convolution of (i) the geometrical source image $(2 \mu \mathrm{m})$, (ii) the vertical pinhole size $(0.5 \mu \mathrm{m})$ and (iii) the diffractionlimited width of the focus for the case of coherent illumination $(140 \mathrm{~nm})$. Assuming Gaussian distributions we can therefore estimate a FWHM of the line focus of about $2.1 \mu \mathrm{m}$. We believe that the discrepancy between the experimental and theoretical line width is mainly caused by deviations from the ideal lens shape. Mask erosion and non-vertically etched side walls lead to the original parabolic mask shape being distorted, which results in lens aberrations and therefore in a broadening of the line focus.

As mentioned previously, all parts of a planar refractive lens have to be more or less perfectly in phase to reach a diffraction-limited spot. If, according to Rayleigh's quarter-wavelength rule (Born \& Wolf, 1999), a phase-mismatch of $\pi / 2$ is tolerable, the energy has to be adjusted with an accuracy $\Delta E / E$ of about $1 / 3000$ for the particular lens that was used to obtain the line focus shown in Fig. 3. The refractive index (which is directly proportional to the density $\delta$ of the diamond) must also be known to this accuracy. This requirement is beyond the capabilities of the density measurements that we performed on our CVD diamond (error $\Delta \delta / \delta \simeq 1 / 200$ ). Actually, in

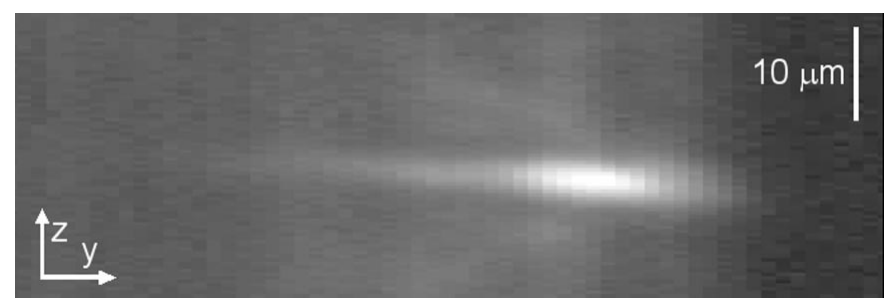

Figure 3

Image of the line focus of a diamond planar refractive lens with $500 \mu \mathrm{m}$ aperture and $1 \mathrm{~m}$ focal length recorded at $17.5 \mathrm{keV}$ photon energy. The vertical FWHM of the obtained spot was $3.2 \mu \mathrm{m}$. the experiment the accuracy requirements were less stringent, as the width of the line focus was strongly determined by other influences (e.g. the size of the geometrical source image, see above). Nevertheless, some of the observed broadening of the line focus may result from a mismatch of the chosen photon energy.

The gain of the lens was calculated from the ratio of the peak intensity in the focal spot to the intensity measured in regions unaffected by the lens. We obtained a gain of 26 from the data shown in Fig. 3. This is about a factor of nine less than would be expected from theory, if we assume an ideal lens in the same experimental situation.

\section{Conclusions}

The present study showed that planar microfabrication technology provides a viable route for the fabrication of refractive diamond lenses that should be capable of withstanding extreme photon flux levels. Although these first results are encouraging, improvement of the patterning process with respect to the obtainable structure height, side-wall roughness and pattern fidelity are needed. A more accurate knowledge of the density and therefore the optical properties of our CVD diamond could completely eliminate a loss in lens performance that is due to a mismatch of the used X-ray energy. Altogether, this should result in a considerable enhancement of the resolution, efficiency and gain that are obtained with such lenses.

We would like to thank B. Patterson and his colleagues for providing the diamond substrates used in this work and M. Kuhnke for letting us use his reactive-ion etcher for the diamond structuring. This work was funded by the Swiss National Science Foundation.

\section{References}

Aristov, V., Grigoriev, M., Kuznetsov, S., Shabelnikov, L., Yunkin, V., Rau, C., Snigirev, A., Snigireva, I., Weitkamp, T., Hoffmann, M. \& Voges, E. (2001). Proc. SPIE, 4145, 285-293.

Aristov, V., Grigoriev, M., Kuznetsov, S., Shabelnikov, L., Yunkin, V., Weitkamp, T., Rau, C., Snigireva, I., Snigirev, A., Hoffmann, M. \& Voges, E. (2000). Appl. Phys. Lett. 77, 4058-4060.

Bionta, R. M. (2000). Technical Report UCRL-ID-137222, January 3, LCLSTN-00-4. Lawrence Livermore National Laboratory, Livermore, CA, USA. Born, M. \& Wolf, E. (1999). Principles of Optics, 7th ed., p. 528. Cambridge University Press.

Chantler, C. T. (1995). J. Phys. Chem. Ref. Data, 24, 71-643.

David, C. \& Souvorov, A. (1999). Rev. Sci. Instrum. 70, 4168-4173.

Freund, A. K. (1995). Opt. Eng. 34, 432-440.

Freund, A. K. (2001). Fundamental and Applied Aspects of Modern Physics, edited by S. H. Connell \& R. Tegen, pp. 135-159. Singapore: World Scientific.

Hasnain, S. S., Kamitsubo, H. \& Mills, D. M. (2001). J. Synchrotron Rad. 8, 1171.

Laclare, J. L. (2001). Nucl. Instrum. Methods Phys. Res. A, 467/468, 1-7.

Lengeler, B. (2002). Private communication.

Lengeler, B., Schroer, C., Tümmler, J., Benner, B., Richwin, M., Snigirev, A., Snigireva, I. \& Drakopoulos, M. (1999). J. Synchrotron Rad. 6, 1153-1167.

Lengeler, B., Schroer, C. G., Benner, B., Günzler, T. F., Kuhlmann, M., Tümmler, J., Simionovici, A. S., Drakopoulos, M., Snigirev, A. \& Snigireva, I. (2001). Nucl. Instrum. Methods Phys. Res. A, 467/468, 944-950.

Lengeler, B., Tümmler, J., Snigirev, A., Snigireva, I. \& Raven, C. (1998). J. Appl. Phys. 84, 5855-5861.

Lund, M. W. (1997). J. X-ray Sci. Tech. 7, 265-270.

Materlik, G. \& Tschentscher, Th. (2001). Editors. The X-ray Free Electron Laser. TESLA Technical Design Report, Part V, Report DESY 2001-01, 
ECFA 2001-209, TESLA Report 2001-23, TESLA-FEL 2001-05, Hamburg, March 2001.

Mills, D. M., Schulte-Schrepping, H. \& Arthur, J. R. (2000). Proc. SPIE, 4143 Nuhn, H. D. et al. (2002). LCLS Conceptual Design Report SLAC-R-593, UC414, Stanford, April 2002. Linac Coherent Light Source, SSRL, Stanford, CA, USA. (http://www-ssrl.slac.stanford.edu/lcls.)

Pantell, R. H., Feinstein, J., Beguiristain, H. R., Piestrup, M. A., Gary, C. K. \& Cremer, J. T. (2001). Proc. SPIE, 4143, 109-117.

Schroer, C. G., Lengeler, B., Benner, B., Kuhlmann, M., Günzler, T. F., Tümmler, J., Rau, C., Weitkamp, T., Snigirev, A. \& Snigireva, I. (2001). Proc. SPIE, 4145, 274-284.

Schroer, C. G., Tümmler, J., Lengeler, B., Drakopoulos, M., Snigirev, A. \& Snigireva, I. (2001). Proc. SPIE, 4143, 60-68.

Schutz, R. J. (1988). VLSI Technology, edited by S. M. Sze. New York: McGraw-Hill.
Snigirev, A., Kohn, V., Snigireva, I. \& Lengeler, B. (1996). Nature (London), 384, 49-50.

Snigireva, I., Snigirev, A., Kutznetsov, S., Rau, C., Weitkamp, T., Shabelnikov, L., Grigoriev, M., Yunkin, V., Hoffman, M. \& Voges, E. (2001). Proc. SPIE, 4499, 64-73.

Sonntag, B. (2001). Nucl. Instrum. Methods Phys. Res. A, 467/468, 8-15.

Tatchyn, R., Arthur, J., Boyce, R., Cremer, T., Fasso, A., Montgomery, J., Vylet, V., Walz, D., Yotam, R., Freund, A. \& Howells, M. R. (1997). Proc. SPIE, 3154, 174-222.

Tatchyn, R. O., Freund, A. K. \& Matsushita, T. (2001). Proc. SPIE, 4500. Tomie, T. (1994). Japanese Patent No. 06045288.

Tomie, T. (1997). US Patent No. 5594773.

Tomie, T. (1998). German Patent No. DE1995019505433.

Wootton, A., Arthur, J., Barbee, T., Bionta, R., London, R., Park, H. S., Ryutov, D., Spiller, E. \& Tatchyn, R. (2001). Proc. SPIE, 4500, 113-122. 